\title{
The effects of substitution of dietary saturated fatty acids with either monounsaturated fatty acids or n-6 polyunsaturated fatty acids on measures of endothelial function, arterial stiffness and blood pressure: results from the DIVAS study
}

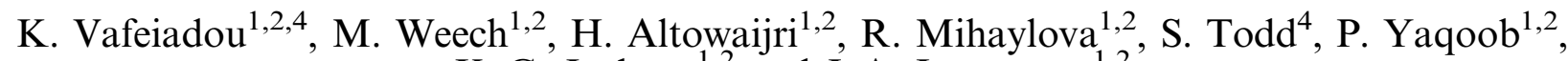 \\ K. G. Jackson ${ }^{1,2}$ and J. A. Lovegrove ${ }^{1,2}$ \\ ${ }^{1}$ Hugh Sinclair Unit of Human Nutrition, ${ }^{2}$ Institute of Cardiovascular and Metabolic Research (ICMR), ${ }^{3}$ Department \\ of Mathematics and Statistics, University of Reading, Reading, RG6 6AP and ${ }^{4}$ School of Life and Medical Sciences, \\ University of Hertfordshire, Hatfield, AL10 9AB
}

Endothelial dysfunction has been recognised as an early modifiable marker in the development of atherosclerosis and risk of cardiovascular disease $(\mathrm{CVD})^{(1)}$. A central public health strategy for the reduction of CVD includes the reduction in dietary saturated fatty acid (SFA) intake ${ }^{(2)}$. However, it remains unclear whether monounsaturated fatty acids (MUFA) or $n-6$ polyunsaturated fatty acids (n-6 PUFA) are the optimal fatty acids to replace dietary SFA. The aim of this study was to determine the effects of substitution of SFA with either MUFA or $n-6$ PUFA on measures of endothelial function, arterial stiffness and blood pressure in men and women at increased risk of developing CVD.

A total of 195 men and women at increased CVD risk (mean age 44 (SD 10) years and BMI 26.9 (SD 4.0) $\mathrm{kg} / \mathrm{m}^{2}$ ) participated in a 16-week, parallel, randomised, controlled, single-blinded intervention study (DIVAS - (Dietary Intervention and VAscular function Study; ClinicalTrials.gov NCT01478958). Participants were randomly assigned (minimised for gender, age, BMI and CVD risk score) to one of the following isoenergetic diets: SFA-rich (target composition: $36 \%$ of total energy ( $\% \mathrm{E}$ ) as total fat, $17 \% \mathrm{E}$ SFA, $11 \%$ E MUFA, 4\%E $n$-6 PUFA), MUFA-rich (36\%E total fat, 9\%E SFA, $19 \%$ E MUFA, 4\%E $n$-6 PUFA), or $n$-6 PUFA-rich $(36 \%$ E total fat, $9 \%$ E SFA, $13 \%$ E MUFA, $10 \%$ E $n-6$ PUFA). A flexible dietary model was developed to deliver the dietary interventions in which exchangeable fats in the habitual diet were replaced by study foods (spreads, oils, snacks) with a specific fatty acid composition. Flow-Mediated Dilatation (FMD) and Laser Doppler imaging (LDI) with iontophoresis were measured for assessing the endothelial function of the macro- and microcirculation, respectively. Pulse Wave Velocity/Analysis (PWV/PWA), Digital Volume Pulse (DVP) (measures of arterial stiffness) and 24-h Ambulatory Blood Pressure (24-h ABP) were also measured at baseline and following 16 weeks of intervention.

A significant deterioration in the FMD response, endothelium-dependent vasodilation of the microvascular circulation (LDI) and mean night SBP and DBP was observed following the SFA-rich diet relative to baseline $(P<0.05)$, but not following either $n-6$ PUFA or MUFA-rich diet. A significant diet interaction was observed for mean night SBP where replacement of SFA with MUFA attenuated the increase observed with SFA $(P<0.05)$

In conclusion, this study showed that dietary SFA had a detrimental effect on vascular reactivity and blood pressure in a group at risk from CVD, which was not observed following the diets rich in unsaturated fatty acids. These data support current public health recommendations to reduce dietary SFA intake as a strategy for CVD risk reduction.

The DIVAS study was supported by the Department of Health and the UK Food Standards Agency (N02044).

1. Widlansky ME, Gokce N, Keaney JF Jr et al. (2003) J Am Coll Cardiol 42, 1149-1160.

2. Astrup A, Dyerberg J, Elwood P et al. (2011) Am J Clin Nutr 93, 684-688. 